\title{
Pressure-dependent Photoluminescence Study of ZnO Nanowires
}

\author{
W. Shan a , W. Walukiewicz, J.W. Ager III, and K.M. Yu \\ Materials Sciences Division, Lawrence Berkeley National Laboratory, Berkeley, CA 94720 \\ Y. Zhang and S.S. Mao \\ Advanced Energy Technologies Department, Environmental Energy Technology Division,, \\ Lawrence Berkeley National Laboratory, Berkeley, CA 94720 \\ R. Kling \\ Department of Semiconductor Physics, University of Ulm, D-89069 Ulm, Germany
}

The pressure dependence of the photoluminescence (PL) transition associated with the fundamental band gap of $\mathrm{ZnO}$ nanowires has been studied at pressures up to $15 \mathrm{GPa}$. $\mathrm{ZnO}$ nanowires are found to have a higher structural phase transition pressure around $12 \mathrm{GPa}$ as compared to $9.0 \mathrm{GPa}$ for bulk $\mathrm{ZnO}$. The pressure-induced energy shift of the near band-edge luminescence emission yields a linear pressure coefficient of $29.6 \mathrm{meV} / \mathrm{GPa}$ with a small sublinear term of $-0.43 \mathrm{meV} / \mathrm{GPa}^{2}$. An effective hydrostatic deformation potential $-3.97 \mathrm{eV}$ for the direct band gap of the $\mathrm{ZnO}$ nanowires is derived from the result.

PACS numbers: 78.67.-n, 78.67.Lt, 62.25.+g

${ }^{\text {a }) ~ E l e c t r o n i c ~ m a i l: ~ W S h a n @ 1 b l . g o v ~}$ 
The fundamental properties of the wide band-gap semiconductor $\mathrm{ZnO}$ have been studied for many years. Recently, there is renewed research interest in $\mathrm{ZnO}$ due to its potential device applications in short-wavelength light emitters and as a transparent conducting material in solar cells. Great progress has been made in the last several years in the synthesis of $\mathrm{ZnO}$ in various forms, particularly on the nanoscale. ${ }^{1-5}$ As a result, room-temperature ultraviolet lasing action in single crystalline $\mathrm{ZnO}$ nanowires was achieved with a much lower threshold owing the superior crystalline quality of the nanowires as compared to the material in bulk form. ${ }^{6}$ It has also been recently found that $\mathrm{ZnO}$ nanocrystals undergo the wurtzite to rocksalt $(\mathrm{NaCl})$ structural transition at a much higher applied pressure $(\sim 15 \mathrm{GPa})$ than that for the bulk $(9.0 \pm 0.5 \mathrm{GPa}){ }^{7}$ Pressure induced hysteresis has also been observed: structurally transformed $\mathrm{ZnO}$ nanocrystals are found to retain the metastable $\mathrm{NaCl}$ phase at ambient conditions. ${ }^{8}$ So far there have been no reported experimental investigations of the optical properties of $\mathrm{ZnO}$ nanocrystals under high pressure to the best of our knowledge.

In this letter, we present the results of a pressure dependent study on the optical properties of single-crystalline $\mathrm{ZnO}$ nanowires. Photoluminescence (PL) measurements were performed in conjunction with the diamond-anvil pressure-cell technique. The strong near bandedge PL emission in the $\mathrm{ZnO}$ nanowires was found to shift almost linearly toward higher energy with increasing pressure. The pressure dependence of both the near band-edge luminescence and a broad mid-gap emission in the nanowire samples were deduced from their energy shift over a wide pressure range. The experimental value of the effective hydrostatic deformation potential of the band gap was also derived from the results.

The $\mathrm{ZnO}$ nanowires were grown with a vapor-liquid-solid process via catalyzed growth, ${ }^{6}$ through which one-dimensional anisotropic crystal growth is promoted by the presence of a 
liquid alloy-solid interface. The first step of growing $\mathrm{ZnO}$ nanowires involves deposition of an $\mathrm{Au}$ thin film on a single crystalline $\mathrm{Si}$ substrate, followed by heating at a temperature approximately $950^{\circ} \mathrm{C}$. Laser evaporation of a $\mathrm{ZnO}$ target was applied in the next step to supply material species so that nucleation and growth of whisker-like $\mathrm{ZnO}$ nanowires are achieved. The diameters of $\mathrm{ZnO}$ nanowires are approximately $100 \mathrm{~nm}$, with lengths in the vicinity of $2 \mu \mathrm{m}$. SEM and TEM images taken from the nanowires are shown in Fig.1.

Photoluminescence measurement was performed at room temperature. The 325-nm line from a $\mathrm{HeCd}$ laser was used as the excitation source. A 1-m double-grating monochromator and a UV-visible photomultiplier tube were used to detect the PL signals. $\mathrm{ZnO}$ nanowires were carefully removed from the substrate and immediately loaded into a gasketed diamond anvil cell which was then sealed using a methanol/ethanol mixture as pressure medium. The standard ruby R1 line method was used for pressure calibration.

Figure 2 shows a PL spectrum taken at atmospheric pressure from 100-nm diameter $\mathrm{ZnO}$ nanowires. The room-temperature luminescence is dominated by strong near-band-edge emission with a peak energy of $3.247 \mathrm{eV}$. The relatively broad linewidth and asymmetric lineshape of the near band-edge emission can be partly attributed to the emission from a series of LO-phonon ( $\sim 72 \mathrm{meV})$ replicas. A weak broadband emission band centered in the green spectral region can also be observed. Under applied pressure, the near band-edge PL emission feature and the broadband emission shift toward higher energy as the $\mathrm{ZnO}$ band gap increases. The intensity of the broad green emission band is found to decrease with pressure. It becomes hardly resolvable at pressure around $10 \mathrm{GPa}$. The near band-edge PL feature does not show any intensity degradation until the applied pressure approaches $12 \mathrm{GPa}$. The inset of Fig.2 shows the near band-edge emission spectra recorded at selected pressures. At pressures above $12 \mathrm{GPa}$, the PL 
intensity decreases rapidly and is completely quenched at 12-15 GPa. Also, at pressures above 12 GPa, the PL peak position deviate significantly from the pressure dependent slope observed at lower pressures. Samples do not show any sign of recovery in terms of luminescence emission even after pressure is reduced down to ambient. A clearly visible change in sample color from white to light yellow indicates a permanent phase transition. These observations are consistent with previous work showing that nanocrystalline $\mathrm{ZnO}$ has its wurtzite to rocksalt phase transition at a higher pressure (15.0 $\mathrm{GPa})$ as compared to the bulk value $(9.0 \pm 0.5 \mathrm{GPa})$ and that pressuretransformed $\mathrm{ZnO}$ is metastable in the PL-inactive rocksalt phase at ambient pressure. ${ }^{7,8}$

The change of the band-edge luminescence peak energy of the $\mathrm{ZnO}$ nanowires together with the green emission band is displayed in Fig. 3 as a function of pressure. The energy positions of the broad green emission band are obtained by fitting the spectral curves to a Gaussian profile. The solid lines in the figure are the least-squares fit to the experimental data using the quadratic-fit function

$$
E(P)=E(0)+\alpha P+\beta P^{2} .
$$

The best fits to the data yield a linear pressure coefficient of $29.6 \mathrm{meV} / \mathrm{GPa}$ with an extremely small sublinear term of $-0.43 \mathrm{meV} / \mathrm{GPa}^{2}$ for the near band-edge emission. The rate of energy shift of the mid-gap green emission with pressure is approximately half of the pressure dependence of the near band-edge emission. A linear slope of $15.9 \mathrm{meV} / \mathrm{GPa}$ and a quadratic term of $-0.71 \mathrm{meV} / \mathrm{GPa}^{2}$ are derived from fitting the experimental date for the green emission, respectively. Since the PL emission at room temperature is associated with the electrons from the conduction-band edge state and the holes in the valence-band edge states, the change of the near band-edge luminescence transition with pressure can be regarded as the dependence of the band gap on pressure for the $\mathrm{ZnO}$ nanowires. The pressure coefficient reported here is somewhat 
larger than those obtained for bulk $\mathrm{ZnO}$ (23.3 to $27.8 \mathrm{meV} / \mathrm{GPa}$ ) using absorption and reflection measurements at pressures up to the structural phase transition at $9.0 \mathrm{GPa} \cdot{ }^{9-11}$

The application of hydrostatic pressure, which causes a shift of the conduction-band edge relative to the valence-band edge due to the change in volume, allows a direct estimation of the effective hydrostatic deformation potential for the $\Gamma$ band gap of wurtzite $\mathrm{ZnO}$. The reason for the term, effective hydrostatic deformation potential is that it is the stress, but not the strain, that is isotropic for the wurtzite structure under hydrostatic pressure. Nevertheless, based on the definition of deformation potential $\boldsymbol{a}=\partial E / \partial n V$, the variation of the band gap with pressure can be appropriately expressed in terms of the relative volume change,

$$
\Delta E=\boldsymbol{a}\left(V-V_{0}\right) / V,
$$

where $V$ is the volume, $V_{0}$ is the volume at atmospheric pressure, $\boldsymbol{a}$ is the hydrostatic deformation potential for the $\Gamma$ band gap. The relative volume change caused by applied pressure can be related by the Murnaghan equation of state: ${ }^{12}$

$$
P=\left(B / B^{\prime}\right)\left[\left(V_{0} / V\right)^{B^{\prime}}-1\right] .
$$

Where $B$ is the bulk modulus of the wurtzite $\mathrm{ZnO}$ and $B^{\prime}$ is its pressure derivative $(=d B / d P)$. By fitting the experimental data to Eq.(3), Using recently reported values of the $\mathrm{ZnO}$ bulk modulus $B=162.4 \mathrm{GPa}$ and its pressure derivative $B^{\prime}=3.6,{ }^{13}$ our result yields an effective hydrostatic deformation potential of $-3.97 \pm 0.15 \mathrm{eV}$ for the direct band gap of $\mathrm{ZnO}$ nanowires. This experimental value of $\boldsymbol{a}$ is comparable to the reported values for the bulk of $-3.51 \sim-3.81 \mathrm{eV}$ and $-3.5 \pm 0.4 \mathrm{eV}$ obtained by different experimental methods. ${ }^{10,11}$

The broad green emission band is commonly observed in the PL spectra of nominally undoped $\mathrm{ZnO}$ single crystals regardless of the crystal growth technique. ${ }^{14-18}$ These results have led to the general belief that the broadband emission in green spectral region involves defects. 
Recent studies on the recombination mechanisms responsible for the green emission in $\mathrm{ZnO}$ phosphors have specifically suggested that the green PL arises from the recombination of electrons in singly occupied oxygen vacancies with photoexcited holes in the valence band. ${ }^{18}$ This assignment is consistent with the weak pressure dependence of the green emission observed here since the initial states involved in the emission are deep localized ones that are much less sensitive to the applied pressure as compared to the direct $\Gamma$ conduction-band edge. Since the energy positions of highly localized states are determined by the electronic states from the whole Brillouin zone, the location of any localized state can be used as the absolute energy reference to separately determine shifts of the conduction and valence band edges. This method has been successfully used to determine the band edge deformation potentials in GaAs and InP. ${ }^{19}$ The assignment of the green emission to the transitions from the localized oxygen vacancy levels to the valence band allows for an estimation of the absolute deformation potentials of conduction and the valence band edges. From the pressure induced shift of the green PL of $15.9 \mathrm{meV} / \mathrm{GPa}$, we obtain the value of $\boldsymbol{a}_{\mathrm{v}} \approx-2.13 \mathrm{eV}$ for the valence band deformation potential. The conduction band deformation potential is then given by $\boldsymbol{a}_{\mathrm{c}}=\boldsymbol{a}+\boldsymbol{a}_{\mathrm{v}}=-6.1 \pm 0.15 \mathrm{eV}$.

In conclusion, the effect of pressure on the luminescence of $\mathrm{ZnO}$ nanowires has been studied. Nanocrystalline $\mathrm{ZnO}$ is found to have a higher structural phase transition pressure around $12 \mathrm{GPa}$ compared to $\sim 9.0 \mathrm{GPa}$ for $\mathrm{ZnO}$ bulk. The pressure induced energy shift of the strong near band-edge PL emission in the samples allows us to determine pressure coefficient for the fundamental band gap of the $\mathrm{ZnO}$ nanowires to be $29.6 \mathrm{meV} / \mathrm{GPa}$ with a small sublinear term of $-0.43 \mathrm{meV} / \mathrm{GPa}^{2}$. Our results also yield the pressure dependence for the broad green emission band with a linear slope of $15.9 \mathrm{meV} / \mathrm{GPa}$ and a quadratic term of $-0.71 \mathrm{meV} / \mathrm{GPa}^{2}$. 
This work is supported by the Director, Office of Science, Office of Basic Energy Sciences, Division of Materials Sciences and Engineering, of the U.S. Department of Energy under Contract No. DE-AC03-76SF00098. 


\section{References}

1. H. Cao, J.Y. Xu, D.Z. Zhang, S.H. Chang, S.T. Ho, E.W. Seelig, X. Liu, R.P.H. Chang, Phys. Rev. Lett. 84, 5584(2000).

2. Y. Li, G.W. meng, L.D. Zhang, and F. Phillipp, Appl. Phys. Lett. 76, 2011(2000).

3. D.M. Bagnall, Y.F. Chen, Z. Zhu, T. Yao, S. Koyama, M.Y. Shen, T. Goto, Appl. Phys. Lett. 70, 2230(1997).

4. M.H. Huang, Y.Y. Wu, H. Feick, N. Tran, E. Weber, and P. Yang, Adv. Materials, 13, 113(2001).

5. Y. Zhang, R. Russo, and S.S. Mao, Appl. Phys. Lett. Submitted.

6. M.H. Huang, S. Mao, H. Feick, H. Yan, Y. Wu, H. Kind, E. Weber, R. Russo, and P. Yang, Science, 292, 1897(2001).

7. J.Z. Jiang, J.S. Oslen, L. Gerward, D. Frost, D. Rubie, and J. Peyronneau, Europhys. Lett. 50, 48(2000).

8. F. Decremps, J. Pellicer_Porres, F. Datchi, J.P. Itiè, A. Polian, F. Baudelet, and J.Z. Jiang, Appl. Phys. Lett. 81, 4820(2002).

9. D.R. Huffmann, L.A. Schwalbe, and D. Schiferl, Solid State Commun. 44, 521(1982).

10. A. Mang, K. Reimann, and St. Rubenacke, Solid State Commun. 94, 251(1995).

11. A. Segura, J.A. Sans, F.J. Manjón, A. Muñoz, and M.J. Herrera-Cabrera, App. Phys. Lett. 83, 278(2003).

12. F.D. Murnaghan, Proc. Natl. Acad. Sci. 30, 244(1944).

13. S. Desgreniers, Phys. Rev. B58, 14102(1998).

14. Ch. Solbrig and E. Rousseau, Solid State Commun. 5, 625(1967). 
15. J.F. Muth, R.M. Kolbas, A.K. Sharma, S. Oktyabrsky, and J. Narayan, J. Appl. Phys. 85, 7884(1999).

16. M.H. Huang, Y. Wu, H. Feick, N. Tran, E. Weber, and P.D. Yang, Adv. Mater. 13, 113(2001).

17. D.A. Lucca, D.W. Hamby, M.J. Kiopfstein, and G. Cantwell, Phys. Stat. Sol. (b) 229, $845(2002)$.

18. K. Vanheusden, W.L. Warren, C.H. Seager, D.R. Tallant, J.A. Voigt, and B.E. Gnade, J. Appl. Phys. 79, 7983(1996).

19. D.D. Nolte, W. Walukiewicz, and E.E. Haller, Phys. Rev. Lett. 59, 501(1987). 


\section{Figure Captions:}

Figure 1. SEM (Top) and TEM (bottom) images of $\mathrm{ZnO}$ nanowires prepared by pulsed laser deposition.

Figure 2. Room-temperature PL spectrum of 100-nm diameter $\mathrm{ZnO}$ nanowires taken at ambient pressure. The inset shows the near band-edge emission at selected pressures.

Figure 3. Effect of pressure on the peak positions of the near band-edge emission and the broad green emission. The solid lines are the best fits to the experimental data. 

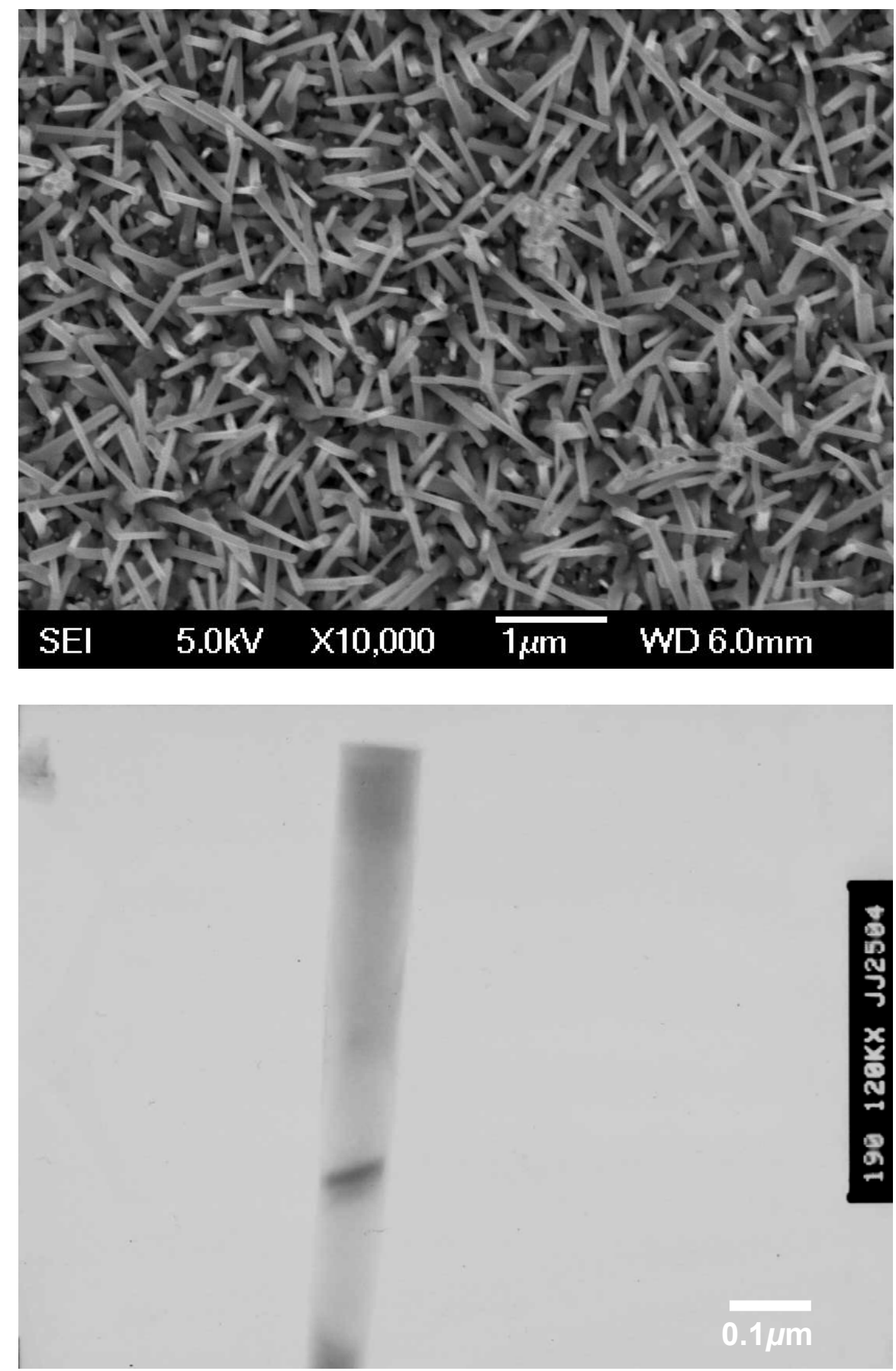

Figure $1 / 3$, Shan et al 


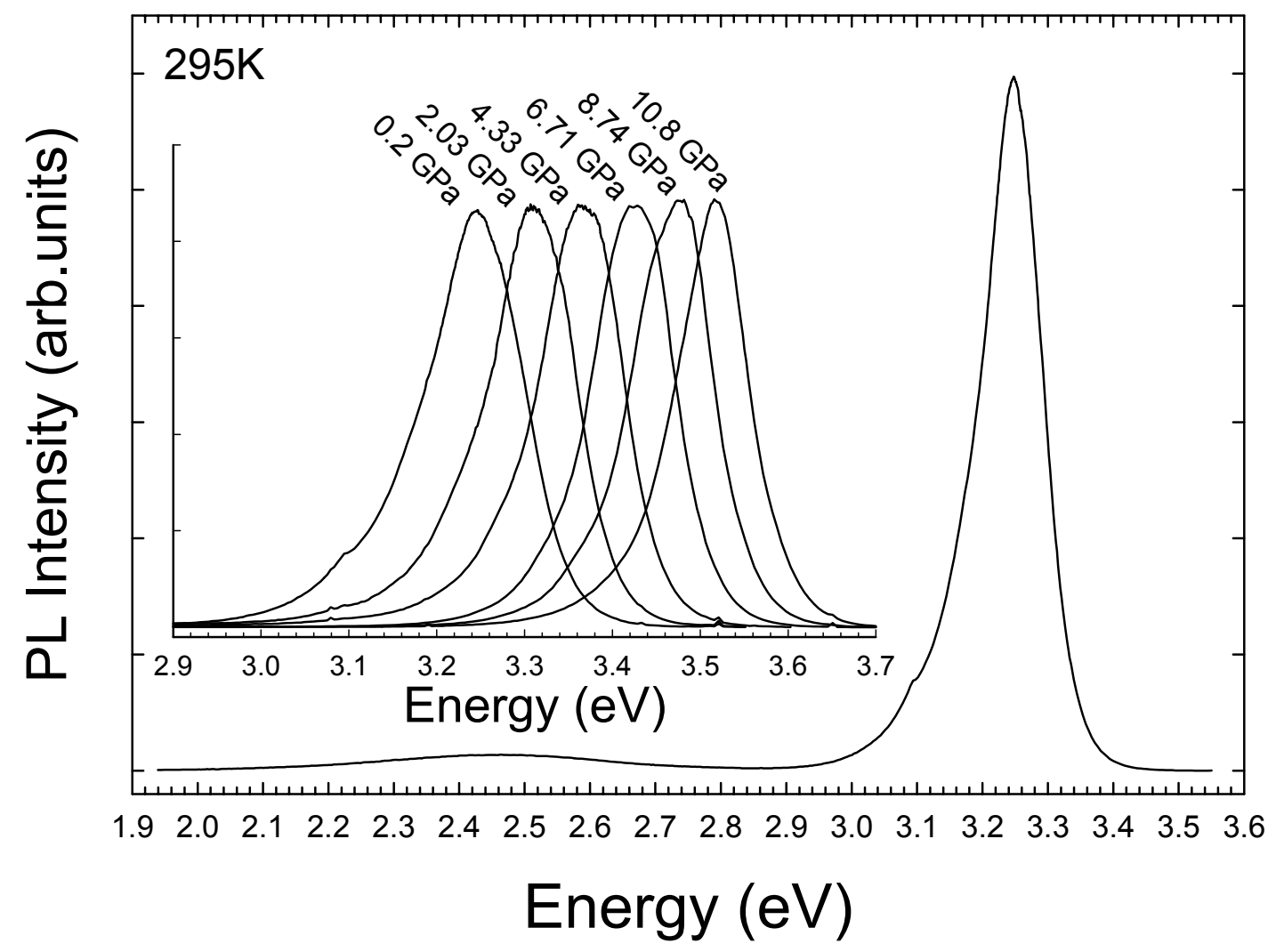

Figure 2/3, Shan et al. 


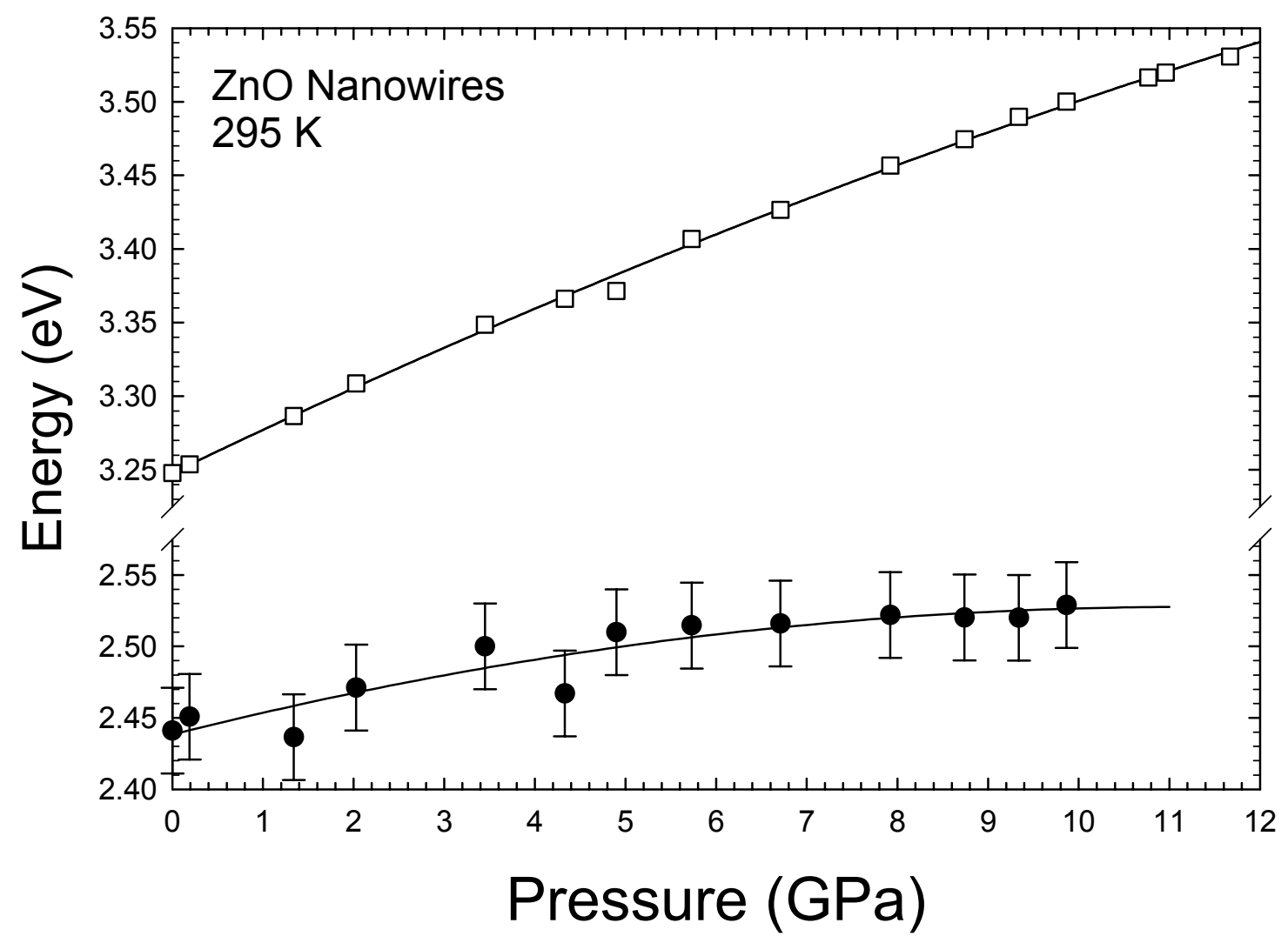

Figure 3/3, Shan et al. 